\title{
Identification of Multivariable Systems with Unknown Feedback Subsystem
}

\author{
Lianming Sun, Hiromitsu Ohmori and Akira Sano \\ Department of System Design Engineering, Keio University \\ 3-14-1 Hiyoshi, Kohoku-ku, Yokohama 223-8522, Japan \\ Email: sun@sano.elec.keio.ac.jp
}

\begin{abstract}
A novel system identification algorithm for a linear discrete-time multivariable closed-loop system is proposed based on an output over-sampling scheme. If the feedback subsystem is operating with period $T$, the structure and parameters of forward and feedback subsystems can be determined simultaneously by using the over-sampled output data, which contain more information about the input to the forward subsystem. It can be used in both control area and social system dynamics even though neither the knowledge of the forward and feedback subsystems are known nor the observation of input to the forward subsystem can be available.
\end{abstract}

\section{Introduction}

Multivariable closed-loop system identification is a very important but rather difficult system identification issue. Ordinarily in the research works associated with such a problem $[1,2,3,4]$, it is often assumed that there is a priori knowledge about the feedback subsystem including its structure and parameters, or the input to the forward subsystem can be observable hence the main identification task is parameter estimation or the structure determination for the forward subsystem dynamics. These assumptions can be satisfied in most of the industry process control systems since the feedback controller are generally designed by the control engineers. However, in some non-technical areas such as environment and economics, various factors or multivariables are connected mutually in a multivariable system models operating with an unknown intrinsic feedback subsystems, and the input to the forward subsystem cannot be available either. Though we can identify some dynamical feature of the entire closed-loop through the recorded data, we cannot distinguish the forward subsystem from the closed-loop, i.e., we cannot identify the closed-loop structure just through the gener- ally recorded input/output data of the closed-loop. It may be one of the most difficult identification tasks in both theory and practice.

On the other hand, some new techniques have been developed to meet the requirement of system analysis, control and prediction. Many researches have demonstrated the effectiveness of a so-called output oversampling scheme in signal processing, blind channel identification $[5,6,7,8]$, etc., and we have also investigated the over-sampling approach to the identification of a closed-loop system which does not satisfy the conventional identifiability conditions [9]. In this paper, we will reveal the effectiveness of the output over-sampling scheme to identification problem of the multivariable closed-loop with unknown feedback.

The paper is organized as follows. A coprime factorization formulation and a state-space model representation of the multivariable system and its closedloop formulation are given first. Next we will investigate the property of the over-sampled forward process dynamics. Then an identification approach using the property of over-sampled model is presented. A numerical simulation example will illustrate the effectiveness of the presented approach.

\section{Problem Statement}

The multivariable closed-loop model considered in this paper is a linear invariant discrete-time model with unknown feedback, which is illustrated in Fig.1. Denote $r$ and $y$ as the input, output of the entire closed-loop system respectively, where $r \in \mathbb{R}^{r}$, $y \in \mathbb{R}^{n}$, and here we assume that $n \geq r$. Furthermore, denote the forward subsystem input as $u$ which has the same dimension as $r$, and the additional interference noise as $w$ which has the same dimension as $y$. In the discrete-time model, we assume that the input signal $r$ has a holding period $T$, and the feedback is operating with period $T$, i.e., only output $y(m T)$ at instant $m T$ is injected into the feedback loop.

In this problem, we assume that the measurable 


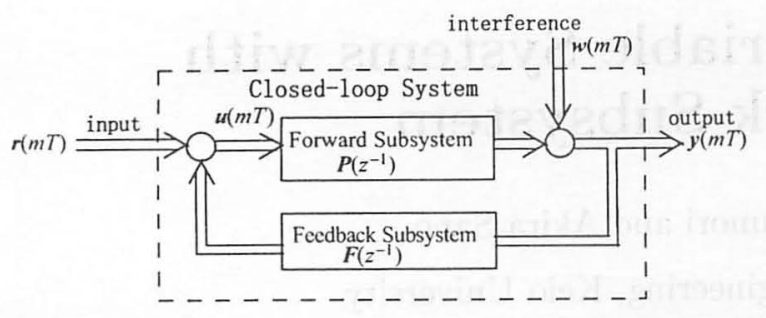

Figure 1. A multivariable discrete-time closed-loop system

signals are only $r$ and $y$, while the input to the forward subsystem, the structure and parameters of the feedback subsystem are not available. The identification task is to determine the forward and feedback subsystems simultaneously just from the recorded data of $r$ and $y$ without the prior knowledge of the feedback. As it has been stated earlier, the forward and feedback subsystems cannot be distinguished from the conventionally observed data $y(m T)$ and $r(m T)$. To identify the forward or feedback subsystem, we over-sample the system output, i.e., we sample the system output with an interval $\Delta$ which is shorter than the input period $T$, and denote the oversampling rate as $p$, where $p=T / \Delta$. The samples of input signal $\boldsymbol{r}$ and output signal $\boldsymbol{y}$ at instant $k \Delta$ are denoted as $r(k \Delta)$ and $y(k \Delta)$ respectively. $w(k \Delta)$ is an i.i.d white noise and is independent of $r$ in this paper.

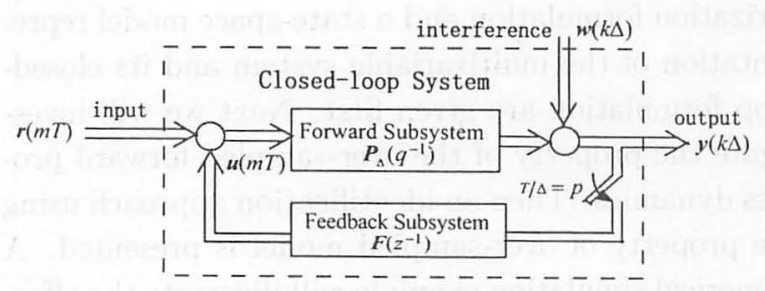

Figure 2. An over-sampled multivariable discretetime closed-loop system

\section{Model Description for Identification}

There are many types of model description can describe a multivariable model. To give the estimation uniquely, the forward and feedback subsystems are described by the following canonical forms.

\subsection{Forward Subsystem Description}

The right coprime factorization form of forward subsystem can be described by

$$
\mathcal{P}: \boldsymbol{y}(m)=\boldsymbol{P}_{R}\left(z^{-1}\right) \boldsymbol{Q}_{R}^{-1}\left(z^{-1}\right) u(m)+w(m)
$$

where $z^{-1}$ is a shift operator and $z^{-1}=e^{-s T}, y(m)$, $u(m)$ and $\boldsymbol{w}(m)$ are the input, output of the forward subsystem, and interference to the system output at instant $m T$ respectively. The subscript " $\mathrm{R}$ " denotes the right coprime factorization. Similarly, the forward subsystem can also be represented by a left coprime factorization, in which the corresponding polynomial matrices are denoted as $\boldsymbol{P}_{L}\left(z^{-1}\right)$ and the left divisor $Q_{L}\left(z^{-1}\right)$.

Another generally used description of multivariable model is a state space representation. For example, the over-sampled model can also be given by

$$
\begin{aligned}
& \boldsymbol{x}(m+1)=\boldsymbol{A} \boldsymbol{x}(m)+\boldsymbol{B} \boldsymbol{u}(m) \\
& \boldsymbol{y}(m)=\boldsymbol{C} \boldsymbol{x}(m) \boldsymbol{D} \boldsymbol{u}(m)+\boldsymbol{w}(m)
\end{aligned}
$$

where $(A, B, C, D)$ are quadruple matrices of the state-space representation.

On the other hand, the over-sampled model with output sampling interval $\Delta$ can be given by

$$
\mathcal{P}_{\Delta}: y_{\Delta}(k)=P_{\Delta, R}\left(q^{-1}\right) Q_{\Delta, R}^{-1}\left(q^{-1}\right) \boldsymbol{u}_{\Delta}(k)+w_{\Delta}(k)
$$

where $q^{-1}$ is a shift operator and $q^{-1}=e^{-s \Delta}$, and $y_{\Delta}(k), u_{\Delta}(k)$ and $w_{\Delta}(k)$ are the forward subsystem output, input and noise at instant $k \Delta$ which are denoted with the subscript of " $\Delta$ " to distinguish from the samples with interval $T$. The state space representation of the over-sampled model can also be given by

$$
\begin{aligned}
& x_{\Delta}(k+1)=\boldsymbol{A}_{\Delta} \boldsymbol{x}_{\Delta}(k)+B_{\Delta} \boldsymbol{u}_{\Delta}(k) \\
& \boldsymbol{y}_{\Delta}(k)=\boldsymbol{C}_{\Delta} \boldsymbol{x}_{\Delta}(k)+\boldsymbol{D}_{\Delta} \boldsymbol{u}_{\Delta}(k)+\boldsymbol{w}_{\Delta}(k)
\end{aligned}
$$

where $\left(A_{\Delta}, B_{\Delta}, C_{\Delta}, D_{\Delta}\right)$ are denoted as quadruple matrices. There are many methods related to the conversion between the state-space description and coprime factorization [10]. It can be seen that the left coprime factorization can be obtained from the observable canonical state-space form, while the right coprime factorization can be obtained from the controllable canonical state-space form.

\subsection{Relation between $\mathcal{P}$ and $\mathcal{P}_{\Delta}$}

Provided that the system output is over-sampled, and the quadruple matrices of a state-space description of $\mathcal{P}_{\Delta}$ are denoted as $\left(A_{\Delta}, B_{\Delta}, C_{\Delta}, D_{\Delta}\right)$. Furthermore, let $m(k)$ be an integer part of the quotient $k / p$, and $j(k)$ be the remainder after devision of $k / p$. For example, if $k=m p+j$, and $k=0, \cdots, p-1$, $m(k)=m, j(k)=j$. Following it, $\boldsymbol{y}_{j}(m)$ and $\boldsymbol{w}_{j}(m)$ are denoted as the system output signal and additional noise at instant $m T+j \Delta$. Furthermore, notice that the input to the forward subsystem $u_{\Delta}(k)$ has holding period $T$ in the over-sampling scheme, then from the state-space representation of the forward subsystem in (4), $\boldsymbol{y}_{j}(m)$ can be repressed by

$$
y_{j}(m)=C_{\Delta}\left(z \boldsymbol{I}-A_{\Delta}^{p}\right)^{-1}\left(z \sum_{i=0}^{j-1} A_{\Delta}^{i} B_{\Delta}\right.
$$




$$
\left.+\sum_{i=j}^{p-1} A_{\Delta}^{i} B_{\Delta}\right) u(m T)+w_{j}(m)
$$

Consider the left coprime factorization formulation of (5). It is noticed that the left divisor in left coprime factorization form is determined by the observable canonical form of matrices pair $\left(A_{\Delta}^{p}, C_{\Delta}\right)$ [10], and $C_{\Delta}\left(z \boldsymbol{I}-\boldsymbol{A}_{\Delta}^{p}\right)^{-1}$ is the common part of (5) for different $j$, then it leads to following results.

Theorem 1 In the over-sampling scheme, let the system input holding period be denoted by $T$, and the system output is sampled with interval $\Delta$ where $\Delta=T / p$, and the over-sampled system model is given in (3). Then the left coprime factorization form of system at $m T+j \Delta$ respecting to period $T$ has the same left divisor $Q_{L}\left(z^{-1}\right)$ for $j=0,1, \cdots, p-1$, and $p$ different left multiplier $\boldsymbol{P}_{L, j}\left(z^{-1}\right)$. And the output $y_{j}(m)$ can be expressed by

$\mathcal{P}_{L, j}: y_{j}(m)=Q_{L}^{-1}\left(z^{-1}\right) P_{L, j}\left(z^{-1}\right) u(m T)+w_{j}(m)$

Furthermore, in (5) we have that

$$
\left(z \boldsymbol{I}-\boldsymbol{A}_{\Delta}^{p}\right)^{-1} \boldsymbol{A}_{\Delta}^{i}=A_{\Delta}^{i}\left(z \boldsymbol{I}-\boldsymbol{A}_{\Delta}^{p}\right)^{-1}
$$

Notice that the right coprime factorization is associated with the controllable canonical form of $\left(A_{\Delta}^{p}, B_{\Delta}\right)$, we also have the following result.

Corollary 1 In the over-sampling scheme, the coprime factorization form of system at $m T+j \Delta$ respecting to period $T$ has the same divisor $Q_{R}\left(z^{-1}\right)$, and $p$ different multiplier $\boldsymbol{P}_{R, j}\left(z^{-1}\right)$ which can be determined from (5) as follows

$\mathcal{P}_{R, j}: y_{j}(m)=P_{R, j}\left(z^{-1}\right) Q_{R}^{-1}\left(z^{-1}\right) u(m T)+w_{j}(m)$

where $j=0, \cdots, p-1$.

Based on Theorem 1 or Corollary 1, the oversampled forward subsystem model can also be described by an MIMO model with $r$ input and $p n$ output in Fig.3, where $\boldsymbol{P}_{j}\left(z^{-1}\right)$ can be expressed by $\mathcal{P}_{L, j}$ in (6) or $\mathcal{P}_{R, j}$ in (7).

Moreover, the coprime factorization of the oversampled system have a following property.

Corollary $2 \bar{Q}\left(q^{-1}\right)=Q_{\Delta, R}^{-1}\left(q^{-1}\right) Q_{R}\left(q^{-p}\right)$ is a polynomial matrix, and $\bar{Q}(0)=I$.

Corollary 3 The coefficients of $P_{\Delta, R}\left(q^{-1}\right)$ respecting to $q^{-1}$ are equal to $P_{R, 1}(0)$.

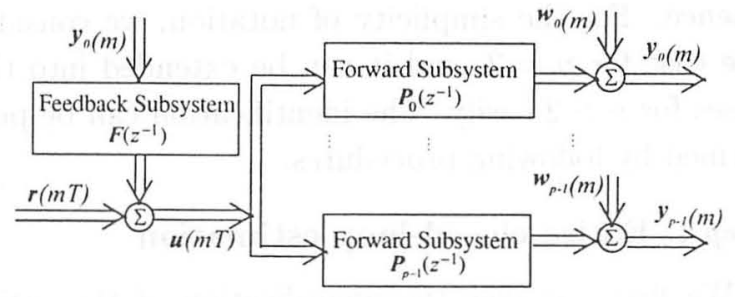

Figure 3. An $p$ MIMO description of the oversampled forward subsystem

\subsection{Closed-loop Description}

Provided that the left coprime factorization form of the feedback subsystem is given by

$$
u(m T)=r(m T)+\boldsymbol{R}_{L}^{-1}\left(z^{-1}\right) S_{L}\left(z^{-1}\right) y(m)
$$

Using the relation described in Fig. $3, \boldsymbol{y}(m)=\boldsymbol{y}_{0}(m)$. Then substituting the right coprime factorization form of $y_{0}(m)$ into (8) will lead to

$$
\begin{aligned}
u(m T)= & Q_{R}\left(z^{-1}\right) \Gamma^{-1}\left(z^{-1}\right)\left(\boldsymbol{R}_{L}\left(z^{-1}\right) \boldsymbol{r}(m T)\right. \\
& \left.+\boldsymbol{S}_{L}\left(z^{-1}\right) \boldsymbol{w}_{0}(m)\right)
\end{aligned}
$$

where

$\Gamma\left(z^{-1}\right)=R_{L}\left(z^{-1}\right) Q_{R}\left(z^{-1}\right)-S_{L}\left(z^{-1}\right) P_{R, 0}\left(z^{-1}\right)$

Then the entire closed-loop system can also be rewritten in the coprime factorization form as follows.

$$
\begin{aligned}
\bar{y}(m) & =\overline{\boldsymbol{P}}_{R}\left(z^{-1}\right) \Gamma^{-1}\left(z^{-1}\right)\left(\boldsymbol{R}_{L}\left(z^{-1}\right) \boldsymbol{r}(m T)\right. \\
& \left.+\boldsymbol{S}_{L}\left(z^{-1}\right) \boldsymbol{w}_{0}(m T)\right)+\overline{\boldsymbol{w}}(m)
\end{aligned}
$$

where

$$
\begin{aligned}
& \overline{\boldsymbol{P}}_{R}^{T}\left(z^{-1}\right)=\left[\begin{array}{lll}
\boldsymbol{P}_{R, 0}^{T}\left(z^{-1}\right) & \cdots & \boldsymbol{P}_{R, p-1}^{T}\left(z^{-1}\right)
\end{array}\right] \\
& \bar{y}^{T}(m)=\left[\begin{array}{lll}
\boldsymbol{y}_{0}^{T}(m) & \cdots & \boldsymbol{y}_{p-1}^{T}(m)
\end{array}\right] \\
& \overline{\boldsymbol{w}}^{T}(m)=\left[\begin{array}{lll}
\boldsymbol{w}_{0}^{T}(m) & \cdots & \boldsymbol{w}_{p-1}^{T}(m)
\end{array}\right]
\end{aligned}
$$

Meanwhile, by regarding $r(m T)$ and $\bar{y}(m)$ as the input and output, the closed-loop in (10) can also be represented by the state-space description of quadruple matrices which are denoted as $\left(A_{c}, B_{c}, C_{c}, D_{c}, E_{c}\right)$ as follows.

$$
\begin{aligned}
& \bar{x}(m+1)=\boldsymbol{A}_{c} \bar{x}(m)+\boldsymbol{B}_{c} \boldsymbol{r}(m)+\boldsymbol{E}_{c} \boldsymbol{w}_{0}(m) \\
& \bar{y}(m)=\boldsymbol{C}_{c} \overline{\boldsymbol{x}}(m)+\boldsymbol{D}_{c} \boldsymbol{r}(m)+\overline{\boldsymbol{w}}(m)
\end{aligned}
$$

By using the results above, we can propose a closedloop structure identification algorithm as follows. 


\section{Identification Algorithm}

Assume that $\boldsymbol{r}(m T)$ is a persistently exciting sequence. For the simplicity of notation, we consider the case for $p=2$, and it can be extended into the cases for $p>2$ easily. The identification can be performed by following procedures.

\section{Step 1: Entire closed-loop estimation}

We first consider the identification of the entire closed-loop. In (10) and (11), if we take $r(m T)$ and $\bar{y}(m)$ as input and output respectively, it can just be regarded as an identification problem of the openloop with colored noise. By adopting MOESP [11] or N4SID [12] combined with instrumental variables, the quadruple system matrices of the entire closed-loop in (11) can be estimated. We denote the estimates as $\hat{\boldsymbol{A}}_{c}, \hat{\boldsymbol{B}}_{c}, \hat{\boldsymbol{C}}_{c}$ and $\hat{\boldsymbol{D}}_{c}$. Then the parameters in (10) can also be calculated from these estimated matrices elements.

\section{Step 2: Estimation of $P_{R}\left(z^{-1}\right)$ and $R_{L}\left(z^{-1}\right)$}

Reviewing (10). Let the right coprime factorization of transfer function $\boldsymbol{\Gamma}^{-1}\left(z^{-1}\right) \boldsymbol{R}_{L}\left(z^{-1}\right)$ be denoted as $\boldsymbol{R}_{R}\left(z^{-1}\right) \Gamma_{R}^{-1}\left(z^{-1}\right)$, and let $\boldsymbol{P}_{R}\left(z^{-1}\right) \boldsymbol{R}_{R}\left(z^{-1}\right)$ be denoted by $\Sigma\left(z^{-1}\right)$. Then (10) can be rewritten as

$$
\begin{aligned}
& \overline{\boldsymbol{y}}(m)=\overline{\boldsymbol{P}}_{R}\left(z^{-1}\right) \boldsymbol{R}_{R}\left(z^{-1}\right) \boldsymbol{\Gamma}_{R}^{-1}\left(z^{-1}\right) \boldsymbol{r}(m T) \\
& \quad+\overline{\boldsymbol{P}}_{R}\left(z^{-1}\right) \boldsymbol{\Gamma}^{-1}\left(z^{-1}\right) \boldsymbol{S}_{L}\left(z^{-1}\right) \boldsymbol{w}_{0}(m)+\overline{\boldsymbol{w}}(m) \\
& =\boldsymbol{\Sigma}\left(z^{-1}\right) \boldsymbol{\Gamma}_{R}^{-1} \boldsymbol{r}(m T)+\overline{\boldsymbol{w}}(m) \\
& \quad+\overline{\boldsymbol{P}}_{R}\left(z^{-1}\right) \boldsymbol{\Gamma}^{-1}\left(z^{-1}\right) \boldsymbol{S}_{L}\left(z^{-1}\right) \boldsymbol{w}_{0}(m)
\end{aligned}
$$

where $\hat{\Sigma}\left(z^{-1}\right)$ and $\hat{\Gamma}_{R}\left(z^{-1}\right)$ can be obtained by converting the estimates of $\hat{\boldsymbol{A}}_{c}, \hat{\boldsymbol{B}}_{c}, \hat{\boldsymbol{C}}_{c}$ and $\hat{\boldsymbol{D}}_{c}$ into right coprime factorization. Next we show that how to separate $\overline{\boldsymbol{P}}_{R}\left(z^{-1}\right)$ from $\boldsymbol{\Sigma}\left(z^{-1}\right)$.

Notice that $\boldsymbol{\Sigma}\left(z^{-1}\right)=\overline{\boldsymbol{P}}_{R}\left(z^{-1}\right) \boldsymbol{R}_{R}\left(z^{-1}\right)$ is the production of $\overline{\boldsymbol{P}}_{R}\left(z^{-1}\right)$ and $\boldsymbol{R}_{R}\left(z^{-1}\right)$. From the property of $z$-transformation, the coefficients of $\Sigma\left(z^{-1}\right)$ can be considered as the convolution of two sequence sets which are composed by the coefficients of $P_{R}\left(z^{-1}\right)$ and $R_{R}\left(z^{-1}\right)$,i.e., the coefficients of $\Sigma\left(z^{-1}\right)$ can be regarded as the output sequence of the multiple FIR filter $\overline{\boldsymbol{P}}_{R}\left(z^{-1}\right)$ with input sequence of the coefficients of $\boldsymbol{R}_{R}\left(z^{-1}\right)$. That is to say that the multiple FIR filter has $r$ input and $p n$ output. Now the problem is how to separate the coefficients of $\bar{P}_{R}\left(z^{-1}\right)$ and $R_{R}\left(z^{-1}\right)$ from $\Sigma\left(z^{-1}\right)$.

This separation problem is very similar to the well known blind channel identification/equalization problem. From the assumption that $n \geq r$, we know that $p n>r$. Then when each row of $P_{R}\left(z^{-1}\right)$ has no common factor, the parameters of $P_{R}\left(z^{-1}\right)$ can be estimated by some blind subspace based identification method [6] or eigen-vector based method
[8] uniquely by respecting $\boldsymbol{R}_{R}(0)=\boldsymbol{I}$. Furthermore, the parameters of $\boldsymbol{R}_{R}\left(z^{-1}\right)$ can be estimated by some well developed deconvolution methods using the estimates of $\boldsymbol{\Sigma}\left(z^{-1}\right)$ and $\boldsymbol{P}_{R}\left(z^{-1}\right)$. By converting $\hat{\boldsymbol{R}}_{R}\left(z^{-1}\right) \hat{\boldsymbol{\Gamma}}_{R}^{-1}\left(z^{-1}\right)$ into the left coprime factorization form, we can obtain $\hat{\boldsymbol{R}}_{L}\left(z^{-1}\right)$ and $\hat{\Gamma}\left(z^{-1}\right)$. Then the remainder problem is the estimation of $Q_{R}\left(z^{-1}\right)$ and $S_{L}\left(z^{-1}\right)$.

\section{Step 3: Estimation of $Q_{R}\left(z^{-1}\right)$ and $S_{L}\left(z^{-1}\right)$}

Consider the closed-loop with input $r_{\Delta}(k)$ and output $\boldsymbol{y}_{\Delta}(k)$ respecting the sampling interval $\Delta$. It is obvious that

$$
\begin{gathered}
y_{\Delta}(k)=P_{\Delta, R}\left(q^{-1}\right) Q_{\Delta, R}^{-1}\left(q^{-1}\right) Q_{R}\left(q^{-p}\right) \Gamma^{-1}\left(q^{-p}\right) \\
\cdot\left(\boldsymbol{R}_{L}\left(q^{-p}\right) r_{\Delta}(k)+S_{L}\left(q^{-p}\right) \overline{\boldsymbol{w}}_{0}(k)\right)+\boldsymbol{w}(k)
\end{gathered}
$$

where $\overline{\boldsymbol{w}}_{0}(k)$ is constructed by holding $\boldsymbol{w}_{o}(m)$ for $T$ as follows.

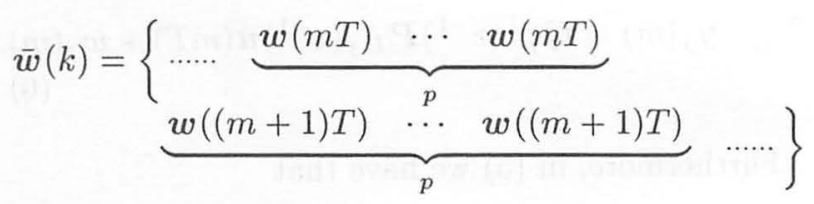

Based on Corollary 2, we know that $\bar{Q}\left(q^{-1}\right)=$ $Q_{\Delta, R}^{-1}\left(q^{-1}\right) Q_{R}\left(q^{-p}\right)$ is a polynomial matrix. Denote $\Lambda\left(q^{-1}\right)=\boldsymbol{P}_{\Delta, R}\left(q^{-1}\right) \bar{Q}\left(q^{-1}\right)$. By using the estimates $\hat{\boldsymbol{R}}_{L}\left(z^{-1}\right)$ and $\hat{\boldsymbol{\Gamma}}\left(z^{-1}\right)$, the entire polynomial matrix of $\Lambda\left(q^{-1}\right)$ can be estimated. Next we deal with two different cases respectively.

(a) Case for $n>r$

Using the blind subspace method given in Step 2, $\hat{\bar{Q}}\left(q^{-1}\right)$ can be separated from the estimate of $\boldsymbol{P}_{\Delta, R}\left(q^{-1}\right) \bar{Q}\left(q^{-1}\right)$ when $n>r$. Since $\bar{Q}\left(q^{-1}\right)=$ $Q_{\Delta}^{-1}\left(q^{-1}\right) Q_{R}\left(q^{-p}\right), \bar{Q}\left(q^{-1}\right)$ can be interpreted as a transfer function matrix with left divisor $Q_{\Delta}^{-1}\left(q^{-1}\right)$. Therefore $\hat{Q}_{R}\left(z^{-1}\right)$ can be determined by following procedures. First transferring the polynomial matrix of $\hat{\bar{Q}}\left(q^{-1}\right)$ into controllable canonical form matrices pair $\left(\hat{\bar{A}}^{(c)}, \hat{\bar{B}}^{(c)}\right)$, and the elements of $\hat{\bar{B}}^{(c)}$ are zeros except the locations of Kronecker invariants are 1. Denote the left divisor of left coprime factorization form of $\left(\left(\hat{\bar{A}}^{(c)}\right)^{p},\left(\hat{\bar{B}}^{(c)}\right)^{T}\right)$ as $\hat{Q}\left(z^{-1}\right)$. Also, from the definition of $\Gamma\left(z^{-1}\right)$, we have that $\hat{Q}_{R}(0)=\hat{\Gamma}(0)$. Then $\hat{Q}_{R}\left(z^{-1}\right)$ can be given by

$$
\hat{Q}_{R}\left(z^{-1}\right)=\hat{Q}\left(z^{-1}\right) \hat{Q}_{R}(0)
$$

Furthermore, denote the sequence $\bar{u}(m T)=$ $Q_{R}^{-1}\left(z^{-1}\right) u(m T)$. Then (7) can be written as

$$
\bar{y}(m)=\bar{P}_{R} \bar{u}(m)+\bar{w}(m)
$$


It can also be considered as an multiple FIR filter with $r$ input $p n$ outputs. Then by using various deconvolution methods such as $[7,8]$, the sequence $\bar{u}(m T)$ can be recovered by using the estimates $\hat{\bar{P}}_{R}^{-1}\left(z^{-1}\right)$. Furthermore, the input sequence $u(m T)$ to the forward subsystem can be estimated by

$$
\hat{u}(m T)=\hat{Q}_{R}\left(z^{-1}\right) \hat{\bar{u}}(m T)
$$

Let the estimate $\hat{S}_{L}\left(z^{-1}\right)$ be satisfied that

$$
\begin{array}{r}
\hat{\boldsymbol{R}}_{L}\left(z^{-1}\right)(\hat{u}(m T)-r(m T)) \\
\quad=\hat{\boldsymbol{S}}_{L}\left(z^{-1}\right) \boldsymbol{y}_{0}(m)+\boldsymbol{\xi}(m)
\end{array}
$$

where $\boldsymbol{\xi}(m)$ can be considered as a noise signal. Then $\hat{S}_{L}\left(z^{-1}\right)$ can be estimated by using PEM [13] or IV [14].

\section{(b) Case for $r=n$}

Premultiplying a nonmodual matrix $\Xi\left(q^{-1}\right)$ to the estimate of $\Lambda\left(q^{-1}\right)=P_{\Delta, R}\left(q^{-1}\right) \bar{Q}\left(q^{-1}\right)$ to satisfy that

$$
\Xi\left(q^{-1}\right) \hat{\Lambda}\left(q^{-1}\right)=q^{-i} \hat{\Pi}\left(q^{-1}\right)
$$

where $i$ is a non-negative integer and the structure of $\hat{\Pi}\left(q^{-1}\right)$ is the same as that of a left divisor of left coprime factorization. Transferring the polynomial matrix of $\hat{\Pi}\left(q^{-1}\right)$ into controllable canonical form matrices $\hat{\bar{A}}^{(c)}$ and $\hat{\bar{B}}^{(c)}$, where the controllable Kronecker invariant indices can be determined by the orders of $\hat{\bar{\Pi}}\left(q^{-1}\right)$. The elements of $\hat{\bar{B}}^{(c)}$ is determined with respecting $\hat{\bar{B}}_{0}^{(c)}=I$, where the rows of $\hat{\bar{B}}_{0}^{(c)}$ are the Kroneker indices rows of $\hat{\bar{B}}^{(c)}$. Denote the left divisor of left coprime factorization of $\left(\left(\hat{\bar{A}}^{(c)}\right)^{p},\left(\hat{\bar{B}}^{(c)}\right)^{T}\right)$ be denoted as $\hat{Q}\left(z^{-1}\right)$, then $\hat{Q}_{R}\left(z^{-1}\right)$ can satisfy that

$$
\hat{\Omega}\left(z^{-1}\right) \hat{Q}_{R}\left(z^{-1}\right)=\hat{Q}\left(z^{-1}\right)
$$

where $\hat{\Omega}\left(z^{-1}\right)$ can be determined later.

Denote the sequence $\bar{u}(m T)=Q_{R}^{-1}\left(z^{-1}\right) u(m)$. Similarly as Case $a, \bar{u}(m T)$ can be recovered by using $\hat{\bar{P}}_{R}\left(z^{-1}\right)$. It can lead to that

$$
\begin{gathered}
\hat{\boldsymbol{Q}}\left(z^{-1}\right) \hat{\bar{u}}(m T)=\hat{\Omega}\left(z^{-1}\right) \boldsymbol{r}(m T)+\hat{\boldsymbol{\Omega}}\left(z^{-1}\right) \\
\cdot \hat{\boldsymbol{R}}_{L}^{-1}\left(z^{-1}\right) \hat{S}_{L}\left(z^{-1}\right) \boldsymbol{y}_{0}(m)+\zeta(m)
\end{gathered}
$$

where $\zeta(m)$ can be considered as a noise signal. Using the estimated $\hat{R}_{L}\left(z^{-1}\right)$, the above estimation problem becomes to an FIR model estimation problem with input $r(m T)$ and $y_{0}(m)$. Then the estimates $\hat{\Omega}\left(z^{-1}\right)$ and $\hat{S}_{L}\left(z^{-1}\right)$ can be obtained. Furthermore, $\hat{Q}_{R}\left(z^{-1}\right)$ can be obtained from (19).

\section{Numerical Simulation}

We deal with a 2 input 3 output closed-loop system whose forward subsystem and feedback subsystem are given by

$$
\begin{aligned}
& \mathcal{P}: \quad P_{R}\left(z^{-1}\right)= {\left[\begin{array}{l}
1.25 z^{-1}-0.3476 z^{-2} \\
2.5 z^{-1}-1.8946 z^{-2} \\
-0.495 z^{-1}-2.0688 z^{-2} \\
2.8 z^{-1}+1.0024 z^{-2} \\
4 z^{-1}+7.3132 z^{-2} \\
8 z^{-1}+5.1687 z^{-2}
\end{array}\right] } \\
& Q_{R}\left(z^{-1}\right)=\left[\begin{array}{c}
1+0.2169 z^{-1}+0.3677 z^{-2} \\
-0.0724 z^{-1}-0.1343 z^{-2} \\
-0.2731 z^{-1}-0.6547 z^{-2} \\
1+0.7006 z^{-1}+0.4703 z^{-2}
\end{array}\right]
\end{aligned}
$$

and

$$
\begin{aligned}
\mathcal{F}: \quad \boldsymbol{R}_{L}\left(z^{-1}\right) & =\left[\begin{array}{cc}
1+0.9338 z^{-1} & -0.6676 z^{-1} \\
0.6478 z^{-1} & 1-0.3371 z^{-1}
\end{array}\right] \\
S_{L}\left(z^{-1}\right) & =\left[\begin{array}{cc}
-0.0340 z^{-1} & -0.0174 z^{-1} \\
-0.0071 z^{-1} & 0.0069 z^{-1} \\
0.0158 z^{-1} & 0.0067 z^{-1}
\end{array}\right]
\end{aligned}
$$

where the feedback subsystem and input signal $r(m T)$ have holding period $T=1.0$. The input signal $r(m T)$ is given as a white sequence with $N(0,1.0)$. Supposed that the system is over-sampled with interval $\Delta=0.5$. The over-sampled forward subsystem with respecting interval $\Delta$ is given by

$$
\begin{aligned}
\mathcal{P}_{\Delta}: \quad P_{\Delta, R}\left(q^{-1}\right)= & {\left[\begin{array}{l}
q^{-1}-0.4674 q^{-2} \\
2 q^{-1}-1.3012 q^{-2} \\
0.5 q^{-1}-1.0789 q^{-2} \\
2 q^{-1}-0.2109 q^{-2} \\
4.9759 q^{-2} \\
4 q^{-1}+1.2463 q^{-2}
\end{array}\right] } \\
Q_{\Delta, R}\left(q^{-1}\right)= & {\left[\begin{array}{c}
1-0.9006 q^{-1}+0.5394 q^{-2} \\
0.0916 q^{-1}-0.0950 q^{-2} \\
0.4880 q^{-1}-0.6232 q^{-2} \\
1-0.7494 q^{-1}+0.6504 q^{-2}
\end{array}\right] }
\end{aligned}
$$

The additional noise $w(k)$ is assumed to be a white noise with $N(0,0.01)$, and is independent of the input $r(m T)$. The observation period is chosen 2000T. The estimation results are as follows:

$$
\hat{P}_{R, 0}\left(z^{-1}\right)=\left[\begin{array}{c}
1.2496 z^{-1}-0.3411 z^{-2} \\
2.4992 z^{-1}-1.8827 z^{-2} \\
-0.4933 z^{-1}-2.0659 z^{-2} \\
2.8008 z^{-1}+0.9831 z^{-2} \\
4.0013 z^{-1}+7.2807 z^{-2} \\
7.9962 z^{-1}+5.1499 z^{-2}
\end{array}\right]
$$




$$
\begin{aligned}
& \hat{\boldsymbol{P}}_{R, 1}\left(z^{-1}\right)=\left[\begin{array}{l}
1.0042+0.1306 z^{-1}-0.2198 z^{-2} \\
1.9969-0.0525 z^{-1}+1.3283 z^{-2} \\
0.4997-2.3219 z^{-1}-0.7386 z^{-2}
\end{array}\right. \\
& 2.0005+1.7130 z^{-1}+0.0672 z^{-2} \\
& 4.0004+7.8425 z^{-1}+1.3043 z^{-2} \\
& 7.9962 z^{-1} \\
& \hat{\boldsymbol{R}}_{L}\left(z^{-1}\right)=\left[\begin{array}{cc}
1+0.9639 z^{-1} & -0.6829 z^{-1} \\
0.6904 z^{-1} & 1-0.3672 z^{-1}
\end{array}\right] \\
& \hat{Q}_{R}\left(z^{-1}\right)=\left[\begin{array}{c}
1+0.2628 z^{-1}+0.3942 z^{-2} \\
-0.0698 z^{-1}-0.1507 z^{-2}
\end{array}\right. \\
& -0.2341 z^{-1}-0.5779 z^{-2} \\
& 1+0.6951 z^{-1}+0.4764 z^{-2} \\
& \hat{\boldsymbol{S}}_{L}\left(z^{-1}\right)=\left[\begin{array}{ll}
-0.0345 z^{-1} & -0.0182 z^{-1} \\
-0.0076 z^{-1} & 0.0064 z^{-1} \\
0.0161 z^{-1} & 0.0072 z^{-1}
\end{array}\right]
\end{aligned}
$$

It can be seen that the estimated results are very closed to the true values.

\section{Conclusion}

In this paper, we have proposed an effective method to identify the forward and feedback subsystems of a multivariable closed-loop system simultaneously. If the feedback subsystem period and the input sequence have holding period $T$, and the output number is not less than the input number, it is demonstrated that the multivariable closed-loop system with an unknown feedback subsystem can be identified respectively by using an over-sampling scheme as though the input to the forward subsystem is unavailable. The identifiability condition for such a complex system and its application to the solution of a practical engineering or social problem are our future research issues.

\section{References}

[1] T. Söderström, L. Ljung and I. Gustavvson, "Identifiability conditions for linear multivariable systems operating under feedback", IEEE Trans. on Automatic Control, Vol.21, No.11, pp.837-840, 1976

[2] S. Niu and D.G. Fisher, "Simultaneous structure identification and parameter estimation of multivariable systems", Int. Journal of Control, Vol.59, No.5, pp.1127-1141, 1994

[3] M. Verhaegen, "Application of a subspace model identification technique to identify LTI systems operating in closed-loop", Automatica, Vol.29, No.4, pp.1029-1040, 1994
[4] P.M.J. Van den Hoff, R.J.P. Schrama, "Identification and control-Closed-loop issues", Automatica, Vol.31, No.12, pp.1751-1770, 1995

[5] L. Tong, G. Xu and T. Kailath, "Blind identification and equalization based on second order statistics: A time domain approach", IEEE Trans. Information Theory, Vol.40, No.2, pp.340-349, 1994.

[6] E. Moulines and P. Duhamel and J. Cardoso and S. Mayrargue, "Subspace methods for the blind identification of multichannel FIR filters", IEEE Trans Signal Processing, Vol.43, No.2, pp.516525,1995

[7] G. Xu and H. Liu and L. Tong and T. Kailath, "A least-squares approach to blind channel identification", IEEE Trans. Signal Processing, Vol.43, No.12, pp.2982-2993, 1995

[8] M.I. Gürelli and C.L. Nikias, "EVAM: An eigenvector-based algorithm for multichannel blind deconvolution of input colored signals", IEEE Trans. Signal Processing, Vol.43, No.1, pp.134-149, 1995

[9] L. Sun, W. Liu and A. Sano, "Over-sampling approach to closed-loop identification", Proc. of the 36th IEEE conference on Decision and Control, pp.1253-1258, San Diego, USA, 1997

[10] T. Kailath, Linear Systems, Prentice-Hall, Englewood Cliffs, N.J., 1980

[11] M. Verhaegen, "Subspace model identification, Part 3. Analysis of the ordinary output-error state-space model identification algorithm", Int. Journal of Control, Vol.58, No.3, pp.555-586, 1993

[12] P. Van Overschee and B. De Moor, "N4SID: Subspace algorithm for the identification of combined deterministic-stochastic systems", Automatica, Vol.30, No.1, pp.75-93, 1994

[13] L. Ljung, Sysyem identification - Theory for the user, Printice Hall PTR, Upper Saddle Reiver, NJ, 1987

[14] T. Söderström and P. Stoica,System identification, Englewood Cliffs, NJ: Prentice Hall, 1989. 